\title{
BMJ Open Lactobacillus reuteri DSM 17938 for managing infant colic: protocol for an individual participant data meta-analysis
}

\author{
Valerie Sung, ${ }^{1}$ Michael D Cabana, ${ }^{2}$ Frank D'Amico, ${ }^{3}$ Girish Deshpande, ${ }^{4,5}$ \\ Christophe Dupont, ${ }^{6}$ Flavia Indrio, ${ }^{7}$ Silja Mentula, ${ }^{8}$ Anna Partty, ${ }^{9}$ \\ Francesco Savino, ${ }^{10}$ Hania Szajewska, ${ }^{11}$ Daniel Tancredi ${ }^{12}$
}

To cite: Sung V, Cabana MD, D'Amico F, et al.

Lactobacillus reuteri DSM 17938 for managing infant colic: protocol for an individual participant data meta-analysis. BMJ Open 2014:4:e006475

doi:10.1136/bmjopen-2014006475

- Prepublication history for this paper is available online To view these files please visit the journal online (http://dx.doi.org/10.1136/ bmjopen-2014-006475).

Received 27 August 2014 Accepted 18 November 2014

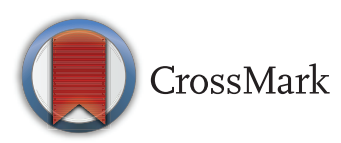

For numbered affiliations see end of article.

Correspondence to Dr Valerie Sung; valerie.sung@rch.org.au

\section{ABSTRACT}

Introduction: Infant colic, or excessive crying of unknown cause in infants less than 3 months old, is common and burdensome. Its aetiology is undetermined, and consensus on its management is still lacking. Recent studies suggest a possible link between infant colic and gut microbiota, indicating probiotics to be a promising treatment. However, only a few strains have been tested, and results from randomised controlled trials are conflicting. It is important to clarify whether probiotics are effective for treating infant colic in general, and to identify whether certain subgroups of infants with colic would benefit from particular strains of probiotics.

Methods and analysis: Through an individual participant data meta-analysis (IPDMA), we aim to identify whether the probiotic Lactobacillus reuteri DSM 17938 is effective in the management of infant colic, and to clarify whether its effects differ according to feeding method (breast vs formula vs combined), proton pump inhibitor exposure, and antibiotic exposure. The primary outcomes are infant crying duration and treatment success (at least $50 \%$ reduction in crying time from baseline) at 21 days postintervention. Individual participant data from all studies will be modelled simultaneously in multilevel generalised linear mixed-effects regression models to account for the nesting of participants within studies. Subgroup analyses of participant-level and intervention-level characteristics will be undertaken on the primary outcomes to assess if the intervention effect differs between certain groups of infants.

Ethics and dissemination: Approved by the Royal Children's Hospital Human Research Ethics Committee (HREC 34081). Results will be reported in a peerreviewed journal in 2015.

Trial registration number: PROSPERO CRD42014013210.

\section{INTRODUCTION}

Infant colic, or excessive crying of unknown cause, is a common, burdensome condition

\section{Strengths and limitations of this study}

- This is the first individual participant data meta-analysis (IPDMA) and the most definitive method to assess the effectiveness of Lactobacillus reuteri DSM 17938 in managing infants with colic, and to clarify which subgroups of infants with colic may benefit from probiotics.

- While individual trials can provide important data, and meta-analyses of randomised controlled trials can give important conclusions, there can be problems with interpreting such conclusions. Combining raw data from individual trials via an IPDMA can yield more reliable estimates of treatment effects with universal applicability. This is particularly important when there is significant chance that particular strains of probiotics may work for particular subgroups of infants with colic, an effect that cannot be detected by individual studies with limited sample sizes.

- The study is limited by the number of participating authors who contribute data to the study, and cannot include data from authors who decline participation. It is also limited by inclusion of studies with potentially different methods of defining infant colic and measuring outcomes.

affecting up to $20 \%$ of infants less than 3 months old. ${ }^{1}$ Although colic self-resolves beyond the first 3 months of life, it is associated with potentially significant adverse effects, such as maternal depression, ${ }^{2}{ }^{3}$ child abuse, ${ }^{4} 5$ and early cessation of breast feeding. ${ }^{6}$ There is also some evidence of long-term adverse outcomes, such as behaviour and sleep problems. ${ }^{78}$ The aetiology of infant colic remains unresolved, and effective treatment options are limited..$^{9-11}$

Recent research has focused on the role of gut microbiota in the pathophysiological 
pathway to infant colic, with numerous studies revealing differences in gut microbiota between infants with and without colic. ${ }^{12-21}$ At the same time, a handful of studies have examined the role of probiotics-live microorganisms believed to confer a health benefit-in the management of infant colic. One study of Lactobacillus reuteri ATCC $55730^{22}$ and two studies of $L$. reuteri DSM $17938^{23} 24$ in breastfed infants with colic were effective, but a subsequent study of both breastfed and formula-fed infants with colic indicated $L$. reuteri DSM 17938 to be ineffective. ${ }^{25}$ Two other studies using different mixtures of probiotic strains were also ineffective in managing colic. ${ }^{1426}$ The reasons for such conflicting evidence are unclear, and there is a need to explore the reasons behind such controversial results, particularly with increasing probiotic marketing, variety of strains used, and addition of probiotics to infant formulae.

Currently, there are some ongoing trials examining the role of probiotics in managing and preventing infant colic, using similar designs, participants, interventions, comparators and outcome measures. ${ }^{27}$ While individual trials can provide important data, and meta-analyses of randomised controlled trials can give important conclusions, there can be problems with interpreting such conclusions. Ultimately, such meta-analyses often do not overcome limitations and biases of individual trials by generating a single best estimate through pooling of treatment effect estimates. ${ }^{28}$ In contrast, combining raw data from individual trials via an individual participant data meta-analysis (IPDMA) can yield more reliable estimates of treatment effects with universal applicability. ${ }^{28-32}$ This is particularly important when there is significant chance that particular strains of probiotics may work for particular subgroups of infants with colic, an effect that cannot be detected by individual studies with limited sample sizes.

The pooling of data into an IPDMA for analysis will ultimately provide more definitive answers as to whether the probiotic $L$. reuteri DSM 17938 is effective for infant colic, and it will also determine whether certain subgroups of infants would benefit from it. As the effects of probiotics are strain specific, ${ }^{33}$ this IPDMA will only include the most commonly studied probiotic strain used for the management of infant colic, and will form the protocol basis for further IPDMAs involving other probiotic strains for the management or prevention of infant colic.

The aims of this IPDMA are:

1. To determine whether the probiotic L. reuteri DSM 17398 is effective in the management of infant colic;

2. To determine whether the effects of $L$. reuteri DSM 17398 on infants with colic differ according to

A. Type of feeding (exclusively breast fed vs partially breast fed vs exclusively formula fed);

B. Proton pump inhibitor exposure;

C. Hypoallergenic formula exposure for formulafed infants;

D. Maternal dairy elimination diets for breastfed infants.

\section{METHODS AND ANALYSIS}

\section{Search methods for identification of studies}

We will search for completed and ongoing randomised controlled trials by identification of published papers and protocols through the online databases MEDLINE, EMBASE, CINAHL, Database of Abstracts of Reviews of Effects (DARE), Cochrane Central Register for Controlled Trials (CENTRAL), and clinical trial registries (eg, metaRegister of Controlled Trials). Reference lists from articles will be explored to identify other potential trials. We will also perform internet searches for non-peer-reviewed articles, media articles and other relevant publications using Google, and approach presenters at relevant conferences and meetings. This IPDMA will be undertaken according to the methods recommended by the Cochrane Collaboration, ${ }^{34}$ with reporting following the preferred reporting items for systematic reviews and meta-analyses (PRISMA) guidelines. $^{35}$

\section{Eligibility criteria for included RCTs}

The IPDMA will include registered randomised controlled trials of the probiotic $L$. reuteri DSM 17398 versus placebo, delivered orally to infants with modified Wessel's definition of infant colic (crying for more than $3 \mathrm{~h}$ of the day, for more than 3 days of the week, for at least 1 week, as recorded by diaries, questionnaires or parental interviews).

Studies evaluating $L$. reuteri ATCC 55730, the mother strain of L. reuteri DSM 17938, will be excluded. L. reuteri ATCC 55730 was found to carry potentially transferable resistance traits for tetracycline and lincomycin. Hence, it was replaced by $L$. reuteri DSM 17938 , a strain without unwanted plasmid-borne resistance. ${ }^{36}$ It remains a matter of debate whether or not $L$. reuteri DSM 17938 , the strain with antibiotic resistance plasmids removed, and the original $L$. reuteri ATCC 55730 strain can be regarded as equal. Moreover, only L. reuteri DSM 17938 is commercially available.

All authors of eligible trials have been contacted and invited to participate in this IPDMA. As more trials satisfying eligibility criteria get published, the relevant authors will be approached and invited to participate, as long as their trials are published within the time frame of conducting this IPDMA.

\section{Main outcomes}

The primary outcomes of the IPDMA are:

- Infant crying duration (minutes per day) at 21 days postintervention;

- Treatment success at 21 days postintervention, defined as at least $50 \%$ reduction in crying time from baseline.

Secondary outcomes include:

- Infant crying duration (minutes per day) at days 7, 14 and 28 postintervention;

- Treatment success (at least 50\% reduction in crying time) at days 7, 14 and 28 postintervention; 
- Infant sleep duration (minutes per day) per $24 \mathrm{~h}$ at $7,14,21$ and 28 days duration (post-treatment baseline);

- Parental report of treatment success, maternal depression, quality of life, and family functioning at the end of the intervention period;

- Adverse effects: diarrhoea, constipation, vomiting, apnoea and apparent life-threatening events (ALTE);

- Stool colonisation analysis;

- Faecal calprotectin levels.

We anticipate that not all included studies will have all secondary outcomes available for analysis, and will analyse only data that are available.

\section{Sample size and power calculation}

Abstracting data from published randomised trials, estimates of the SDs in crying time (min/day) at baseline and day 21 were collected and pooled to provide an estimated SD of 210 (min/day). From this information, it is estimated that approximately 120 infants per treatment group would be sufficient for detecting a mean difference in treatment groups of $80 \mathrm{~min} /$ day (power $=0.80$, $\alpha=0.05$, two-tailed). Additionally, approximately 120 per group would also provide $80 \%$ power for detecting a difference of 20 percentage points $(\alpha=0.05$, two-tailed $)$ in the treatment success rates. Treatment success is defined as (yes/no) with 'yes' corresponding to at least 50\% reduction in crying time from baseline to day 21.

For subgroup analysis to compare whether treatment effects differed by patient characteristics, hypothesis testing will be based on the comparison of treatment effects between subgroups, with a two-tailed $\alpha$ of 0.10 used to offset the decreased precision available for estimating interaction effects (ie, differences in differences). We specified that it would be clinically significant to detect between-subgroup differences in treatment effects of $150 \mathrm{~min} /$ day on the crying time outcome and of 50 percentage points on the treatment success outcome, assuming that one subgroup consists of between $33 \%$ and $66 \%$ of the full sample and the other subgroup consists of the remainder. For example, if treatment group differences truly are $180 \mathrm{~min} /$ day in a prespecified subgroup with one-third of the patients and only $30 \mathrm{~min} /$ day for the remaining patients, the difference in treatment effects would correspond to $150 \mathrm{~min} /$ day. Again, a sample size of approximately 120 infants provides at least $80 \%$ power to detect such clinically important differences.

At the time of this writing, authors of four trials have agreed to participate. These four trials include three that assessed $L$. reuteri strain DSM 17398 , comprising a total of 293 patients with 150 randomised to probiotic and 143 to placebo. Thus, it is projected that this IPDMA will have sufficient power for detecting clinically relevant differences in both the average crying times and success rates of at least $50 \%$ reduction from baseline to day 21 between the probiotic and placebo groups.

\section{Statistical analysis}

The analysis will be conducted with individual participant data from all studies modelled simultaneously in multilevel generalised linear mixed-effects regression models to account for the nesting of participants within studies. ${ }^{29}$ Models will be specified with fixed-effects terms for the individual participant's binary indicator treatment-assignment (probiotic vs control), a parsimonious set of prespecified participant-level characteristics, and the study identifier. This model specification will be straightforwardly extended to account for when longitudinally assessed outcomes are the units of analysis (one record per time point per participant), by including fixed-effects terms for time (main effects as well as interaction terms with the binary treatment indicator) and random effects for the participant to account for residual within-participant correlation. Standard choices of link and variance functions will be specified, according to type of outcome, with linear-normal models used for suitably (ie, homogeneous) continuous outcomes and logit-binomial and log-Poisson models used for binary and count outcomes, respectively.

Subgroup analyses of participant-level and intervention-level characteristics will be undertaken on the primary outcomes to assess if the intervention effect differs between certain groups of infants. Heterogeneity of treatment effects will be formally assessed by respecifying regression models with interaction terms for the binary treatment indicator with the candidate-effect modifier and conducting formal hypothesis testing (with a statistical significance threshold reset to 0.10 to help offset the low statistical power associated with testing interaction terms). These characteristics are identified a priori and include: (1) feeding method (exclusively breast fed vs partially breast fed vs exclusively formula fed), (2) proton pump inhibitor exposure, (3) hypoallergenic formula exposure for formula-fed infants and (4) maternal dairy elimination diets for breast-fed infants. Confounders identified a priori will include (1) family history of atopy, (2) delivery type (vaginal vs caesarean), (3) enrolment age and (4) antibiotic use.

Analysis will be by intention-to-treat; specifically, the binary treatment term will correspond to assigned treatment. Missing data patterns and assumptions will be described, and the potential impact of alternative missing data assumptions on the primary analysis will be explored in sensitivity analyses. The primary analysis will be conducted at the 0.05 level of significance.

\section{ETHICS AND DISSEMINATION}

\section{Ethics committee approval}

The IPDMA has been approved by the Royal Children's Hospital Human Research Ethics Committee (HREC 34081). The IPDMA is registered at PROSPERO, the International Prospective Register of Systematic Reviews, at the University of York (CRD42014013210). 


\section{Project management and data collection}

Membership to the collaboration for this IPDMA will include representatives from each trial contributing data to the project, a project coordination team, and a data management team consisting of two independent statisticians (FDA and DT). The collaboration will collect the minimum de-identified data required to answer the research questions. We will store data in a secure, centralised, customised database, accessible only by a unique passcode known only to the project coordination team, data management team, and managers of each individual study contributing data. The two independent statisticians will inspect the data with respect to range, internal consistency, and missing items by checking them against published reports, trial protocols and, if necessary, data collection sheets. The statisticians will discuss any inconsistencies or missing data with individual trial managers, and any problems will be resolved by consensus using original raw data.

\section{Data ownership and confidentiality}

All included trials must have been given ethical approval by their respective HREC. Participants in individual trials must have consented to their participation in their respective trial. Each study manager remains the custodian of their own data and retains the right to withdraw their data from the analysis at any time. Data must be de-identified before being shared for this IPDMA. The pooled data can be accessed by the project coordination team, data management team, and managers of each individual study contributing data. The project intellectual property (IP) will be owned by the parties as tenants in common in proportion to their respective contributions to that project IP (including, without limitation, contributions and inventorship).

\section{Data monitoring procedures}

Each individual trial will follow its own data monitoring procedures. The collaboration plans to update the IPDMA data at regular intervals if further relevant individual trials are completed with available data.

\section{Risks and benefits}

The main risk for this study is the discovery of discrepant data, or results that are inconsistent with published manuscripts; however, all the studies have been published in peer-reviewed, scientific journals. In addition, this risk will be minimised by careful handling of the data, involvement of two independent statisticians in data analysis, having a unified plan for management of missing data, and the plan for open discussions to resolve any issues regarding any conflicting information. Participation in this study requires prior consent and approval of all trial managers in sharing each study's data and subjecting individual data to reanalysis. There is also a risk of inadequate representation of all trial participants due to authors who do not consent to their data being pooled into the IPDMA.
The combination of data from multiple, similar trials via an IPDMA can yield more reliable estimates of treatment effects, especially for small subgroups of patients. ${ }^{29-32}$ The patients enrolled in each of the individual studies may have had particular patient characteristics or exposures that may have affected the effectiveness of the probiotic intervention. In addition, the different studies may have varied in the types of patients they recruited or varied slightly in their recruitment criteria. As a result, in addition to making more definitive conclusions as to whether probiotics are effective for infant colic, this IPDMA will be able to help determine if there are subgroups of infants who might benefit from a probiotic intervention for colic, in general, or to a particular probiotic strain.

\section{Publication plan}

Each individual trial will have the right to publish its main results before publication of this IPDMA. Study results from this meta-analysis will be reported in a peerreviewed journal in 2015. Before publication of any IPDMA manuscripts, drafts will be circulated for comment, revision and approval by a nominated representative of each of the participating trials. Publications using these data will be authored on behalf of the IPDMA collaboration, with specific named authors (including a representative of each participating trial, the project coordination team and data management team) according to the amount of contribution to each manuscript, and names of other participating collaborators listed in the Acknowledgements.

\section{STRENGTHS AND LIMITATIONS OF THE STUDY}

This is the first IPDMA and the most definitive method to assess the effectiveness of $L$. reuteri DSM 17938 in managing infants with colic, and to clarify which subgroups of infants with colic may benefit from probiotics. The study is limited by the number of participating authors who contribute data to the study, and cannot include data from authors who decline participation. The study is also limited by inclusion of studies with differing methods of defining infant colic and measuring outcomes. The collaboration formed through this IPDMA will be the platform to conduct future IPDMAs for the probiotic management and prevention of infant colic.

\section{Author affiliations}

${ }^{1}$ Murdoch Childrens Research Institute, Royal Children's Hospital, The University of Melbourne, Melbourne, Victoria, Australia

${ }^{2}$ Department of Pediatrics, Epidemiology and Biostatistics, University of California, San Francisco (UCSF) School of Medicine, San Francisco,

California, USA

${ }^{3}$ Duquesne University/UPMC St. Margaret Hospital, Pittsburgh, Pennsylvania, USA

${ }^{4}$ Department of Children's health division and NICU, Nepean Hospital Sydney, Sydney, New South Wales, Australia

${ }^{5}$ Sydney Medical School Nepean, University of Sydney, Sydney, New South Wales, Australia 
${ }^{6}$ Department of Pediatric Gastroenterology, Paris Descartes University, Necker Hospital, Paris, France

${ }^{7}$ Department of Pediatrics, University of Bari, Bari, Italy

${ }^{8}$ Bacteriology Unit, National Institute for Health and Welfare, Helsinki, Finland

${ }^{9}$ Department of Pediatrics and Adolescent Medicine, Turku University Hospital, Turku, Finland

${ }^{10}$ Ospedale Infantile Regina Margherita, Città della Salute e della Scienza di Torino, Torino, Italy

${ }^{11}$ Department of Paediatrics, The Medical University of Warsaw, Warsaw, Poland

${ }^{12}$ Department of Pediatrics, UC Davis Health System, Sacramento, California, USA

Acknowledgements The authors thank Mary Ellen Sanders for organising all funding arrangements from ISAPP and for organising the ISAPP meeting in Aberdeen, UK in June 2014

Contributors VS, MDC, FDA and DT conceptualised the study protocol. VS and MDC are the project coordinators of this collaboration. FDA and DT are the data managers of this study. All authors contributed to the design of the study protocol. VS produced the first draft of the manuscript. All authors contributed to the writing of the manuscript and read and approved the final manuscript.

Funding This work was supported by the International Scientific Association for Probiotics and Prebiotics (ISAPP). ISAPP has contributed to the costs of all teleconferences and has facilitated the first meeting for this collaboration in June 2014 in Aberdeen, UK. ISAPP will also offer support for the statistical work involved in this IPDMA. ISAPP has contributed to the fees for submission of this manuscript for publication. Each individual trial has received funding from their own respective funding bodies. VS is supported by a National Health and Medical Research Council Postgraduate Scholarship 607447. The Murdoch Childrens Research Institute is supported by the Victorian Government's Operational Infrastructure Support Program.

Competing interests VS, MDC, FDA, GD, FI, SM, FS, HS and DT have received travel reimbursement to attend the ISAPP meeting in Aberdeen, UK in June 2014. MDC is a board member of the ISAPP and has received grant funding from Nestec; he has served as a paid consultant for Nestle, Mead Johnson and Pfizer Nutrition. FDA and DT will receive funding from ISAPP for their work in the statistical analysis. In the past 5 years, DT has also received travel reimbursement to attend annual ISAPP meetings (2009-2014) and scientific consulting fees (2012).HS and FI served as speakers for BioGaia, the manufacturer of $L$. reuteri DSM 17938. CD received honoraria from Sodilac for a clinical trial. ${ }^{26} \mathrm{FS}$ reports receiving a travel grant from Nestlè Italy; personal fees from Mead Johnson Nutrition, Italy; personal fees from Cana S.A.S. Thessaloniki, Greece; personal fees from Nutricia-Part of Group Danone, Dubai Kuwait; travel grants and other from BioGaia AB, Stockholm I Sweden; personal fees from HiPP GmbH and Co Vertrieb KG Germany; a travel grant from Nestlé France SAS, Paris; travel grants and other from Noos, srl, Roma Italy; personal fees from A. MENARINI IFR s.r.I, Firenze Italy outside the submitted work.

Ethics approval The Royal Children's Hospital Human Research Ethics Committee.

Provenance and peer review Not commissioned; externally peer reviewed.

Open Access This is an Open Access article distributed in accordance with the Creative Commons Attribution Non Commercial (CC BY-NC 4.0) license, which permits others to distribute, remix, adapt, build upon this work noncommercially, and license their derivative works on different terms, provided the original work is properly cited and the use is non-commercial. See: http:// creativecommons.org/licenses/by-nc/4.0/

\section{REFERENCES}

1. Lucassen PL, Assendelft WJ, van Eijk JT, et al. Systematic review of the occurrence of infantile colic in the community. Arch Dis Child 2001;84:398-403.

2. McMahon C, Barnett B, Kowalenko N, et al. Postnatal depression, anxiety and unsettled infant behaviour. Aust N Z J Psychiatry 2001;35:581-88.
3. Smart J, Hiscock H. Early infant crying and sleeping problems: a pilot study of impact on parental well-being and parent-endorsed strategies for management. J Paediatr Child Health 2007; 43:284-90.

4. Barr RG, Trent RB, Cross J. Age-related incidence curve of hospitalized Shaken Baby Syndrome cases: convergent evidence for crying as a trigger to shaking. [see comment]. Child Abuse Negl 2006;30:7-16.

5. Lee C, Barr RG, Catherine N, et al. Age-related incidence of publicly reported shaken baby syndrome cases: is crying a trigger for shaking? J Dev Behav Pediatr 2007;28:288-93.

6. Howard CR, Lanphear N, Lanphear BP, et al. Parental responses to infant crying and colic: the effect on breastfeeding duration. Breastfeed Med 2006;1:146-55.

7. Savino $\mathrm{F}$, Castagno E, Bretto R, et al. A prospective 10-year study on children who had severe infantile colic. Acta Paediatr Suppl 2005;94:129-32.

8. Hemmi MH, Wolke D, Schneider S. Associations between problems with crying, sleeping and/or feeding in infancy and long-term behavioural outcomes in childhood: a meta-analysis. Arch Dis Child 2011;96:622-9.

9. Garrison MM, Christakis DA. A systematic review of treatments for infant colic. [see comment]. Pediatrics 2000;

106(1 Pt 2):184-90.

10. Lucassen PL, Assendelft WJ. Systematic review of treatments for infant colic. Pediatrics 2001;108:1047-8.

11. Cohen-Silver J, Ratnapalan S. Management of infantile colic: a review. Clinical Pediatr (Phila) 2009;48:14-17.

12. Lehtonen L, Korvenranta $H$, Eerola $E$. Intestinal microflora in colicky and noncolicky infants: bacterial cultures and gas-liquid chromatography. J Pediatr Gastroenterol Nutr 1994; 19:310-14.

13. de Weerth $C$, Fuentes $S$, Puylaert $P$, et al. Intestinal microbiota of infants with colic: development and specific signatures. Pediatrics 2013;131:e550-8.

14. Mentula S, Tuure T, Koskenala R, et al. Microbial composition and fecal fermentation end products from colicky infants-a probiotic supplementation pilot. Microb Ecol Health Dis 2008;20:37-47.

15. Partty A, Kalliomaki M, Endo A, et al. Compositional development of Bifidobacterium and Lactobacillus microbiota is linked with crying and fussing in early infancy. PLoS One 2012;7:e32495.

16. Rhoads JM, Fatheree NY, Norori J, et al. Altered fecal microflora and increased fecal calprotectin in infants with colic. J Pediatr 2009;155:823-28.e1.

17. Roos S, Dicksved J, Tarasco V, et al. 454 pyrosequencing analysis on faecal samples from a randomized DBPC trial of colicky infants treated with Lactobacillus reuteri DSM 17938. PLoS One 2013;8: e56710.

18. Savino F, Bailo E, Oggero R, et al. Bacterial counts of intestinal Lactobacillus species in infants with colic. Pediatr Allergy Immunol 2005;16:72-5.

19. Savino F, Cordisco L, Tarasco V, et al. Molecular identification of coliform bacteria from colicky breastfed infants. Acta Paediatr 2009;98:1582-8.

20. Savino F, Cordisco L, Tarasco V, et al. Antagonistic effect of Lactobacillus strains against gas-producing coliforms isolated from colicky infants. BMC Microbiol 2011;11:157.

21. Savino F, Cresi F, Pautasso S, et al. Intestinal microflora in breastfed colicky and non-colicky infants. Acta Paediatr 2004;93:825-9.

22. Savino $\mathrm{F}$, Pelle $\mathrm{E}$, Palumeri $\mathrm{E}$, et al. Lactobacillus reuteri (American Type Culture Collection Strain 55730) versus simethicone in the treatment of infantile colic: a prospective randomized study. Pediatrics 2007;119:e124-30.

23. Savino F, Cordisco L, Tarasco V, et al. Lactobacillus reuteri DSM 17 938 in infantile colic: a randomized, double-blind, placebo-controlled trial. Pediatrics 2010;126:e526-33.

24. Szajewska H, Gyrczuk E, Horvath A. Lactobacillus reuteri DSM 17938 for the management of infantile colic in breastfed infants: a randomized, double-blind, placebo-controlled trial. J Pediat 2013;162:257-62.

25. Sung V, Hiscock $\mathrm{H}$, Tang $\mathrm{M}$, et al. Treating infant colic with the probiotic Lactobacillus reuteri: a randomised double-blind placebo-controlled trial. Bmj 2014;348:g2107.

26. Dupont C, Rivero M, Grillon C, et al. Alpha-lactalbumin-enriched and probiotic supplemented infant formula in infants with colic: growth and gastrointestinal tolerance. Eur J Clin Nutr 2010; 64:767.

27. metaRegister of Controlled Trials. Registered trials numbers ISRCTN95287767, NCT01067027, NCT01279265, NCT01541046, NCT01017991, NCT00167700, NCT01036243, NCT00893711, 
NCT01849991. 2014. http://wwwcontrolled-trialscom/mrct/searchhtml (accessed 29 Jul 2014).

28. Berlin JA, Golub RM. Meta-analysis as evidence: building a better pyramid. JAMA 2014;312:603-5.

29. Riley RD, Lambert PC, Abo-Zaid G. Meta-analysis of individual participant data: rationale, conduct, and reporting. Bmj 2010;340:c221.

30. Tugwell P, Knottnerus JA. Advantages of individual patient data analysis in systematic reviews. J Clin Epidemiol 2010;63:233-4.

31. Soll RF. Individual patient meta-analysis in pediatrics. Pediatrics 2011;128:775-6.

32. van Walraven $\mathrm{C}$. Individual patient meta-analysis-rewards and challenges. J Clin Epidemiol 2010;63:235-7.
33. Rowland I, Capurso L, Collins $\mathrm{K}$, et al. Current level of consensus on probiotic science-report of an expert meeting-London, 23 November 2009. Gut Microbes 2010;1:436-9.

34. Higgins JPT, Green S (eds). Cochrane Handbook for Systematic Reviews of InterventionsVersion 5.1.0 [updated March 2011]. The Cochrane Collaboration, 2011. www.cochrane-handbook.org.

35. Moher D, Liberati A, Tetzlaff J, et al. Preferred Reporting Items for Systematic Reviews and Meta-Analyses: the PRISMA statement. $J$ Clin Epidemiol 2009;62:1006-12.

36. Rosander A, Connolly E, Roos S. Removal of antibiotic resistance gene-carrying plasmids from Lactobacillus reuteri ATCC 55730 and characterization of the resulting daughter strain, L. reuteri DSM 17938. Appl Environ Microbiol 2008;74:6032-40. 